New integral inequalities involving beta function via $P$-convexity

\author{
Wenjun Liu
}




\title{
NEW INTEGRAL INEQUALITIES INVOLVING BETA FUNCTION VIA $P$-CONVEXITY
}

\author{
WENJUN LIU \\ Received 05 December, 2012
}

\begin{abstract}
In this note we establish some estimates, involving the Euler Beta function, of the integral $\int_{a}^{b}(x-a)^{p}(b-x)^{q} f(x) d x$ for functions when a power of the absolute value is $P$-convex. An extension to functions of several variables is also obtained.
\end{abstract}

2010 Mathematics Subject Classification: 33B15, 26D15, 26B25

Keywords: Euler Beta function, integral inequality, Hölder's inequality, $P$-convexity

\section{INTRODUCTION}

Let $I$ be an interval in $\mathbb{R}$. Then $f: I \rightarrow \mathbb{R}$ is said to be convex if

$$
f(t x+(1-t) y) \leq t f(x)+(1-t) f(y)
$$

holds for all $x, y \in I$ and $t \in[0,1]$.

The notion of quasi-convex functions generalizes the notion of convex functions. More precisely, a function $f:[a, b] \rightarrow \mathbb{R}$ is said to be quasi-convex on $[a, b]$ if

$$
f(t x+(1-t) y) \leq \max \{f(x), f(y)\}
$$

holds for any $x, y \in[a, b]$ and $t \in[0,1]$. Clearly, any convex function is a quasiconvex function. Furthermore, there exist quasi-convex functions which are not convex (see [11]).

The generalized quadrature formula of Gauss-Jacobi type has the form

$$
\int_{a}^{b}(x-a)^{p}(b-x)^{q} f(x) d x=\sum_{k=0}^{m} B_{m, k} f\left(\gamma_{k}\right)+\mathcal{R}_{m}[f]
$$

for certain $B_{m, k}, \gamma_{k}$ and rest term $\mathcal{R}_{m}[f]$ (see [22]).

In [17], Özdemir et al. established several integral inequalities concerning the left-hand side of (1.1) via some kinds of convexity. Especially, they discussed the following result connecting with quasi-convex function: 
Theorem 1. Let $f:[a, b] \rightarrow \mathbb{R}$ be continuous on $[a, b]$ such that $f \in L([a, b])$, $0 \leq a<b<\infty$. If $f$ is quasi-convex on $[a, b]$, then for some fixed $p, q>0$, we have

$$
\int_{a}^{b}(x-a)^{p}(b-x)^{q} f(x) d x \leq(b-a)^{p+q+1} \beta(p+1, q+1) \max \{f(a), f(b)\},
$$

where $\beta(x, y)$ is the Euler Beta function.

Recently, Liu [12] established some new integral inequalities for quasi-convex functions as follows:

Theorem 2. Let $f:[a, b] \rightarrow \mathbb{R}$ be continuous on $[a, b]$ such that $f \in L([a, b])$, $0 \leq a<b<\infty$ and let $k>1$. If $|f|^{\frac{k}{k-1}}$ is quasi-convex on $[a, b]$, for some fixed $p, q>0$, then

$$
\begin{aligned}
& \int_{a}^{b}(x-a)^{p}(b-x)^{q} f(x) d x \\
\leq & (b-a)^{p+q+1}[\beta(k p+1, k q+1)]^{\frac{1}{k}}\left(\max \left\{|f(a)|^{\frac{k}{k-1}},|f(b)|^{\frac{k}{k-1}}\right\}\right)^{\frac{k-1}{k}} .
\end{aligned}
$$

Theorem 3. Let $f:[a, b] \rightarrow \mathbb{R}$ be continuous on $[a, b]$ such that $f \in L([a, b])$, $0 \leq a<b<\infty$ and let $l \geq 1$. If $|f|^{l}$ is quasi-convex on $[a, b]$, for some fixed $p, q>0$, then

$$
\begin{aligned}
& \int_{a}^{b}(x-a)^{p}(b-x)^{q} f(x) d x \\
\leq & (b-a)^{p+q+1} \beta(p+1, q+1)\left(\max \left\{|f(a)|^{l},|f(b)|^{l}\right\}\right)^{\frac{1}{l}} .
\end{aligned}
$$

On the other hand, Dragomir et al. in [6] defined the following class of functions of $P$-convex.

Definition 1. Let $I \subseteq \mathbb{R}$ be an interval. The function $f: I \rightarrow \mathbb{R}$ is said to belong to the class $P(I)$ (or to be $P$-convex) if it is nonnegative and, for all $x, y \in I$ and $t \in[0,1]$, satisfies the inequality

$$
f(t x+(1-t) y) \leq f(x)+f(y) .
$$

Note that $P(I)$ contain all nonnegative convex and quasiconvex functions. Since then numerous articles have appeared in the literature reflecting further applications in this category; see $[1,2,4,5,7-10,13-16,18-21,23-26]$ and references therein.

The main purpose of this note is to establish some new estimates of the integral $\int_{a}^{b}(x-a)^{p}(b-x)^{q} f(x) d x$ for functions when a power of the absolute value is $P$-convex. An extension to functions of several variables is also obtained. That is, this study is a continuation and further generalization of $[12,17]$ via $P$-convexity. 


\section{NeW INTEgRAL INEQUALITIES VIA $P$-CONVEXITy}

In this section we generalize Theorems $1-3$ with a $P$-convex function setting. For this purpose, we need the following lemma (see [17, Lemma 2.1]):

Lemma 1. Let $f:[a, b] \subset[0, \infty) \rightarrow \mathbb{R}$ be continuous on $[a, b]$ such that $f \in$ $L([a, b]), a<b$. Then the equality

$$
\int_{a}^{b}(x-a)^{p}(b-x)^{q} f(x) d x=(b-a)^{p+q+1} \int_{0}^{1}(1-t)^{p} t^{q} f(t a+(1-t) b) d t
$$

holds for some fixed $p, q>0$.

The next theorem gives a new result for $P$-convex functions.

Theorem 4. Let $f:[a, b] \rightarrow \mathbb{R}$ be continuous on $[a, b]$ such that $f \in L([a, b])$, $0 \leq a<b<\infty$. If $|f|$ is $P$-convex on $[a, b]$, for some fixed $p, q>0$, then

$$
\begin{aligned}
& \int_{a}^{b}(x-a)^{p}(b-x)^{q} f(x) d x \\
\leq & (b-a)^{p+q+1} \beta(p+1, q+1)(|f(a)|+|f(b)|),
\end{aligned}
$$

where $\beta(x, y)$ is the Euler Beta function.

Proof. By Lemma 1, the Beta function which is defined for $x, y>0$ as

$$
\beta(x, y)=\int_{0}^{1} t^{x-1}(1-t)^{y-1} d t
$$

and the fact that $f$ is $P$-convex on $[a, b]$, we have

$$
\begin{aligned}
\int_{a}^{b}(x-a)^{p}(b-x)^{q} f(x) d x & \leq(b-a)^{p+q+1} \int_{0}^{1}(1-t)^{p} t^{q}|f(t a+(1-t) b)| d t \\
& \leq(b-a)^{p+q+1} \int_{0}^{1}(1-t)^{p} t^{q}(|f(a)|+|f(b)|) d t \\
& =(b-a)^{p+q+1} \beta(q+1, p+1)(|f(a)|+|f(b)|)
\end{aligned}
$$

which completes the proof.

The corresponding version for powers of the absolute value is incorporated in the following result.

Theorem 5. Let $f:[a, b] \rightarrow \mathbb{R}$ be continuous on $[a, b]$ such that $f \in L([a, b])$, $0 \leq a<b<\infty$ and let $k>1$. If $|f|^{\frac{k}{k-1}}$ is $P$-convex on $[a, b]$, for some fixed $p, q>0$, then

$$
\int_{a}^{b}(x-a)^{p}(b-x)^{q} f(x) d x
$$




$$
\leq(b-a)^{p+q+1}[\beta(k p+1, k q+1)]^{\frac{1}{k}}\left(|f(a)|^{\frac{k}{k-1}}+|f(b)|^{\frac{k}{k-1}}\right)^{\frac{k-1}{k}} .
$$

Proof. By Lemma 1, Hölder's inequality, the definition of Beta function and the fact that $|f|^{\frac{k}{k-1}}$ is $P$-convex on $[a, b]$, we have

$$
\begin{aligned}
& \int_{a}^{b}(x-a)^{p}(b-x)^{q} f(x) d x \\
\leq & (b-a)^{p+q+1}\left[\int_{0}^{1}(1-t)^{k p} t^{k q} d t\right]^{\frac{1}{k}}\left[\int_{0}^{1}|f(t a+(1-t) b)|^{\frac{k}{k-1}} d t\right]^{\frac{k-1}{k}} \\
\leq & (b-a)^{p+q+1}[\beta(k q+1, k p+1)]^{\frac{1}{k}}\left[\int_{0}^{1}\left(|f(a)|^{\frac{k}{k-1}}+|f(b)|^{\frac{k}{k-1}}\right) d t\right]^{\frac{k-1}{k}} \\
= & (b-a)^{p+q+1}[\beta(k q+1, k p+1)]^{\frac{1}{k}}\left(|f(a)|^{\frac{k}{k-1}}+|f(b)|^{\frac{k}{k-1}}\right)^{\frac{k-1}{k}},
\end{aligned}
$$

which completes the proof.

A more general inequality using Lemma 1 is as follows:

Theorem 6. Let $f:[a, b] \rightarrow \mathbb{R}$ be continuous on $[a, b]$ such that $f \in L([a, b])$, $0 \leq a<b<\infty$ and let $l>1$. If $|f|^{l}$ is $P$-convex on $[a, b]$, for some fixed $p, q>0$, then

$$
\begin{aligned}
& \int_{a}^{b}(x-a)^{p}(b-x)^{q} f(x) d x \\
\leq & (b-a)^{p+q+1} \beta(p+1, q+1)\left(|f(a)|^{l}+|f(b)|^{l}\right)^{\frac{1}{l}} .
\end{aligned}
$$

Proof. By Lemma 1, Hölder's inequality, the definition of Beta function and the fact that $|f|^{l}$ is $P$-convex on $[a, b]$, we have

$$
\begin{aligned}
& \int_{a}^{b}(x-a)^{p}(b-x)^{q} f(x) d x \\
= & (b-a)^{p+q+1} \int_{0}^{1}\left[(1-t)^{p} t^{q}\right]^{\frac{l-1}{l}}\left[(1-t)^{p} t^{q}\right]^{\frac{1}{l}} f(t a+(1-t) b) d t \\
\leq & (b-a)^{p+q+1}\left[\int_{0}^{1}(1-t)^{p} t^{q} d t\right]^{\frac{l-1}{l}}\left[\int_{0}^{1}(1-t)^{p} t^{q}|f(t a+(1-t) b)|^{l} d t\right]^{\frac{1}{l}} \\
\leq & (b-a)^{p+q+1}[\beta(q+1, p+1)]^{\frac{l-1}{l}}\left[\left(|f(a)|^{l}+|f(b)|^{l}\right) \beta(q+1, p+1)\right]^{\frac{1}{l}} \\
= & (b-a)^{p+q+1} \beta(p+1, q+1)\left(|f(a)|^{l}+|f(b)|^{l}\right)^{\frac{1}{l}},
\end{aligned}
$$

which completes the proof. 


\section{AN EXTENSION TO FUNCTIONS OF SEVERAL VARIABLES}

In this section some new integral inequalities for functions of several variables on convex subsets of $\mathbb{R}^{n}$ will be given. First we recall the notion of $P$-convexity for functions on a convex subset $U$ of $\mathbb{R}^{n}$.

Definition 2 ([3, Definition 3.1]). The function $f: U \rightarrow \mathbb{R}$ is said to be $P$-convex on $U$ if it is nonnegative and, for all $x, y \in U$ and $\lambda \in[0,1]$, satisfies the inequality

$$
f(\lambda x+(1-\lambda) y) \leq f(x)+f(y) .
$$

The following proposition will be used throughout this section.

Proposition 1 ([3, Proposition 3.2]). Let $U \subseteq \mathbb{R}$ be a convex subset of $\mathbb{R}$ and $f: U \rightarrow \mathbb{R}$ be a function. Then $f$ is $P$-convex on $U$ if and only if, for every $x, y \in U$, the function $\varphi:[0,1] \rightarrow \mathbb{R}$, defined by

$$
\varphi(t):=f((1-t) x+t y),
$$

is $P$-convex on $I$ with $I=[0,1]$.

We have the following inequalities for functions of several variables on convex subsets of $\mathbb{R}^{n}$.

Theorem 7. Let $U \subseteq \mathbb{R}$ be a convex subset of $\mathbb{R}$. Assume that $f: U \rightarrow \mathbb{R}^{+}$is a $P$-convex function on $U$. Then, for every $x, y \in U$ and every $[a, b] \in[0,1]$ with $a<b$, the following inequality holds:

$$
\begin{aligned}
& \int_{a}^{b}(t-a)^{p}(b-t)^{q} f((1-t) x+t y) d t \\
\leq & (b-a)^{p+q+1} \beta(p+1, q+1)[f((1-a) x+a y)+f((1-b) x+b y)] .
\end{aligned}
$$

Proof. Let $x, y \in U$ and every $[a, b] \in[0,1]$ with $a<b$. Since $f: U \rightarrow \mathbb{R}^{+}$is a $P$-convex function, by Proposition 1 the function $\varphi:[0,1] \rightarrow \mathbb{R}^{+}$defined by

$$
\varphi(t):=f((1-t) x+t y),
$$

is $P$-convex on $I$ with $I=[0,1]$. Applying Theorem 4 to the function $\varphi$ implies that

$$
\begin{aligned}
& \int_{a}^{b}(t-a)^{p}(b-t)^{q} \varphi(t) d t \\
\leq & (b-a)^{p+q+1} \beta(p+1, q+1)(|\varphi(a)|+|\varphi(b)|),
\end{aligned}
$$

and we deduce that (3.1) holds.

Similarly, we have 
Theorem 8. Let $U \subseteq \mathbb{R}$ be a convex subset of $\mathbb{R}$ and let $k>1$. Assume that $f \frac{k}{k-1}: U \rightarrow \mathbb{R}^{+}$is a $P$-convex function on $U$. Then, for every $x, y \in U$ and every $[a, b] \in[0,1]$ with $a<b$, the following inequality holds:

$$
\begin{gathered}
\int_{a}^{b}(t-a)^{p}(b-t)^{q} f((1-t) x+t y) d t \\
\leq(b-a)^{p+q+1}[\beta(k p+1, k q+1)]^{\frac{1}{k}} \\
{\left[f^{\frac{k}{k-1}}((1-a) x+a y)+f^{\frac{k}{k-1}}((1-b) x+b y)\right]^{\frac{k-1}{k}} .}
\end{gathered}
$$

Theorem 9. Let $U \subseteq \mathbb{R}$ be a convex subset of $\mathbb{R}$ and let $l>1$. Assume that $f^{l}$ : $U \rightarrow \mathbb{R}^{+}$is a $P$-convex function on $U$. Then, for every $x, y \in U$ and every $[a, b] \in$ $[0,1]$ with $a<b$, the following inequality holds:

$$
\begin{aligned}
& \int_{a}^{b}(t-a)^{p}(b-t)^{q} f((1-t) x+t y) d t \\
\leq & (b-a)^{p+q+1} \beta(p+1, q+1)\left[f^{l}((1-a) x+a y)+f^{l}((1-b) x+b y)\right]^{\frac{1}{l}} .
\end{aligned}
$$

\section{ACKNOWLEDGEMENT}

This work was partly supported by the National Natural Science Foundation of China (Grant No. 11301277), the Qing Lan Project of Jiangsu Province, and the Teaching Research Project of NUIST (Grant No. 12JY052, 13ZYKC01).

\section{REFERENCES}

[1] A. O. Akdemir and M. E. Özdemir, "Some Hadamard-type inequalities for coordinated $P$-convex functions and Godunova-Levin functions," AIP Conf. Proc., vol. 1309, pp. 7-15, 2010.

[2] M. Alomari, M. Darus, and S. S. Dragomir, "Inequalities of Hermite-Hadamard's type for functions whose derivatives absolute values are quasi-convex," RGMIA Res. Rep. Coll., vol. 12, p. 11, Article 14, 2009.

[3] A. Barani and S. Barani, "Hermite-Hadamard type inequalities for functions when a power of the absolute value of the first derivative is P-convex," Bull. Aust. Math. Soc., vol. 86, no. 1, pp. 126-134, 2012.

[4] S. S. Dragomir, "On some new inequalities of Hermite-Hadamard type for $m$-convex functions," Tamkang J. Math., vol. 33, no. 1, pp. 55-65, 2002.

[5] S. S. Dragomir and C. E. M. Pearce, Selected topics on Hermite-Hadamard inequalities and applications, ser. RGMIA Monographs. Victoria University, 2000.

[6] S. S. Dragomir, J. E. Pečarić, and L. Persson, "Some inequalities of Hadamard type," Soochow J. Math., vol. 21, no. 3, pp. 335-341, 1995.

[7] S. Dragomir and C. Pearce, "Quasi-convex functions and Hadamard's inequality," Bull. Aust. Math. Soc., vol. 57, no. 3, pp. 377-385, 1998.

[8] A. Házy, "Bernstein-Doetsch type results for $h$-convex functions," Math. Inequal. Appl., vol. 14, no. 3, pp. 499-508, 2011.

[9] A. Házy, "Bernstein-Doetsch type results for $(k, h)$-convex functions," Miskolc Math. Notes, vol. 13, no. 2, pp. 325-336, 2012. 
[10] V. N. Huy and Nguyen Thanh Chung, "Some generalizations of the Fejér and Hermite-Hadamard inequalities in Hölder spaces," J. Appl. Math. Inform., vol. 29, no. 3-4, pp. 859-868, 2011.

[11] D. A. Ion, "Some estimates on the Hermite-Hadamard inequality through quasi-convex functions," An. Univ. Craiova, Ser. Mat. Inf., vol. 34, pp. 83-88, 2007.

[12] W. Liu, "New integral inequalities via $(\alpha, m)$-convexity and quasi-convexity," Hacet. J. Math. Stat., vol. 42, no. 3, pp. 289-297, 2013.

[13] W. Liu, "Some Ostrowski type inequalities via Riemann-Liouville fractional integrals for $h$ convex functions," J. Comput. Anal. Appl., vol. 16, no. 5, pp. 998-1004, 2014.

[14] W. Liu, "Some Simpson type inequalities for $h$-convex and $(\alpha, m)$-convex functions," J. Comput. Anal. Appl., vol. 16, no. 5, pp. 1005-1012, 2014.

[15] B. Micherda and T. Rajba, "On some Hermite-Hadamard-Fejér inequalities for $(k, h)$-convex functions," Math. Inequal. Appl., vol. 15, no. 4, pp. 931-940, 2012.

[16] M. E. Özdemir and C. Yildiz, "New inequalities for Hermite-Hadamard and Simpson type and applications," arXiv, no. 1103.1965v1 [math.CA].

[17] M. E. Özdemir, E. Set, and M. Alomari, "Integral inequalities via several kinds of convexity," Creat. Math. Inform., vol. 20, no. 1, pp. 62-73, 2011.

[18] M. Z. Sarikaya, A. Saglam, and H. Yildirim, "On some Hadamard-type inequalities for $h$-convex functions," J. Math. Inequal., vol. 2, no. 3, pp. 335-341, 2008.

[19] M. Z. Sarikaya, E. Set, and M. Ozdemir, "On some new inequalities of Hadamard type involving h-convex functions," Acta Math. Univ. Comen., New Ser., vol. 79, no. 2, pp. 265-272, 2010.

[20] E. Set, M. Sardari, M. E. Özdemir, and J. Rooin, "On generalizations of the Hadamard inequality for $(\alpha, m)$-convex functions," RGMIA Res. Rep. Coll., vol. 12, p. 10, Article 4, 2009.

[21] E. Set, M. Özdemir, and S. S. Dragomir, "On Hadamard-type inequalities involving several kinds of convexity," J. Inequal. Appl., vol. 2010, p. 12, 2010.

[22] D. D. Stancu, G. Coman, and P. Blaga, Analiză numerică şi teoria aproximării. Vol. II. ClujNapoca: Presa Universitară Clujeană, 2002.

[23] K. L. Tseng, G. S. Yang, and S. S. Dragomir, "On quasiconvex functions and Hadamard's inequality," RGMIA Res. Rep. Coll., vol. 6, no. 3, p. 12, Article 1, 2003.

[24] M. Tunç, "On some integral inequalities via $h$-convexity," Miskolc Math. Notes, vol. 14, no. 3, pp. 1041-1057, 2013.

[25] M. Tunç, "Ostrowski-type inequalities via $h$-convex functions with applications to special means," J. Inequal. Appl., vol. 2013, p. 10, 2013.

[26] S. Varošanec, “On h-convexity,” J. Math. Anal. Appl., vol. 326, no. 1, pp. 303-311, 2007.

Author's address

Wenjun Liu

College of Mathematics and Statistics, Nanjing University of Information Science and Technology, Nanjing 210044, China

E-mail address: wjliu@nuist.edu.cn 